\title{
Unexpected Reactivity switch in the Statistical Copolymerization of 2- Oxazolines and 2-Oxazines Enabling the One-Step Synthesis of Amphiphilic Gradient Copolymers
}

Ondrej Sedlacek, ${ }^{\mathrm{a}}$ Kathleen Lava, ${ }^{\mathrm{a}}$ Bart Verbraeken, ${ }^{\mathrm{a}}$ Sabah Kasmi, ${ }^{\mathrm{b}}$ Bruno G. De Geest ${ }^{\mathrm{b}}$ and Richard Hoogenboom ${ }^{\mathrm{a}, *}$

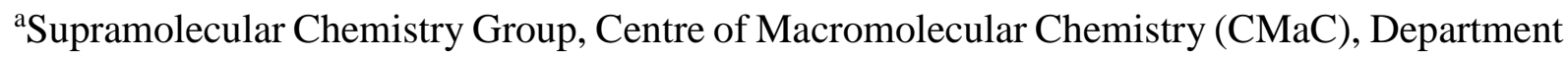
of Organic and Macromolecular Chemistry, Ghent University, Krijgslaan 281 S4, B-9000 Ghent, Belgium.

${ }^{\mathrm{b}}$ Department of Pharmaceutics, Ghent University, B-9000 Ghent, Belgium

E-mail: richard.hoogenboom@ugent.be 


\section{Materials}

2-Methyl-2-oxazine (MeOzi), 2-n-propyl-2-oxazoline (PrOx) and 2-n-butyl-2-oxazoline $(\mathrm{BuOx})$ were prepared using literature procedure ${ }^{1}$ and were distilled from $\mathrm{BaO}$ before use. Methyl $p$-toluenesulfonate (MeOTs) was obtained from Sigma-Aldrich and was distilled from $\mathrm{CaH}_{2}$ before use. Acetonitrile (Sigma-Aldrich) was purified over aluminum oxide using a solvent purification system from J.C. Meyer. All other chemicals, including octadecyl rhodamine, curcumin and phosphate buffer-saline tablets were purchased from Sigma-Aldrich and were used as received. Water was deionized with a Millipore Milli-Q water purification system.

\section{Instrumentation}

All stock solutions and samples were prepared in a VIGOR Sci-Lab SG 1200/750 glovebox system with a water concentration $\leq 0.1 \mathrm{ppm}$. For the polymerizations, a Biotage Initiator EXP microwave system with Robot Sixty was used. Gas chromatography $(G C)$ was used to monitor the kinetics of the CROP employing an Agilent 7890A system equipped with a VWR Carrier160 hydrogen generator and an Agilent HP-5 column of $30 \mathrm{~m}$ length and $0.32 \mathrm{~mm}$ diameter. An FID detector was used, and the inlet was set to $240{ }^{\circ} \mathrm{C}$ with a split injection ratio 25:1. Hydrogen was used as carrier gas at a flow rate of $2 \mathrm{~mL} \mathrm{~min}^{-1}$ Size exclusion chromatography (SEC) was used to determine the molecular weights $\left(M_{w}\right.$ - mass-averaged molecular weight, $M_{n}$ - number-averaged molecular weight) and the dispersity $\left(\nexists=M_{m} / M_{n}\right)$ of the prepared polymers. This was performed using an Agilent 1260-series HPLC system equipped with a 1260 ISO-pump, a 1260 automatic liquid sampler, a thermostatted column compartment at $50{ }^{\circ} \mathrm{C}$ equipped with two PLgel $5 \mu \mathrm{m}$ mixed-D columns and a precolumn in series, a 1260 diode array detector 1260 RI detector and multi-angle light scattering detector (Wyatt miniDawn Treos II). The used eluent was DMA containing $50 \mathrm{mM}$ of $\mathrm{LiCl}$ at a flow rate of 0.5

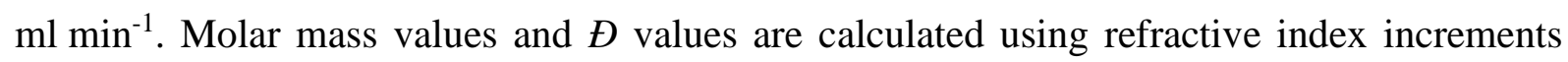
$d n / d c_{\mathrm{PMeOzi}}=0.081$ and $d n / d c_{\mathrm{PPrOx}}=0.074$ and $d n / d c_{\mathrm{PBuOx}}=0.051$, respectively. The refractive index increments of copolymers were calculated as weighed molar averages $d n / d c$ of individual homopolymers. Nuclear magnetic resonance (NMR) spectra were measured with a Bruker Advance MSL $400 \mathrm{MHz}$ NMR spectrometer. All chemical shifts are given in ppm. Turbidity measurements were used to determine the cloud point temperature $\left(T_{\mathrm{CP}}\right)$ of the polymers in phosphate buffer-saline (PBS, $\mathrm{pH}=7.4, c_{\mathrm{pol}}=5 \mathrm{mg} \mathrm{mL}^{-1}$ ) using Crystal 16 parallel crystallizer 
turbidimeter (Avantium Technologies) connected to a recirculation chiller at a heating rate of $0.5^{\circ} \mathrm{C} \mathrm{min}{ }^{-1}$. The $T_{\mathrm{CP}}$ was reported as the temperature with $50 \%$ transmittance in the heating run. Dynamic light scattering (DLS) measurements were used to measure hydrodynamic diameters $\left(D_{h}\right)$ of prepared polymers using a Zetasizer NanoZS instrument, Model ZEN3600 (Malvern Instruments, UK). The polymer samples were prepared in PBS by direct dissolution in the same manner as for the turbidity measurements and were filtered before measurement through an $0.22 \mu \mathrm{m}$ PTFE filter. Due to the possible multimodal peak distribution, the volumeweighted mean $D_{h}$ was determined at a scattering angle of $\theta=173^{\circ}$ and the DTS (Nano) program was used to evaluate the data. The volume distribution was derived from the intensity distribution using Mie theory. After each increase in temperature $\left(0.5^{\circ} \mathrm{C}\right.$ step $)$, the sample was equilibrated for $4 \mathrm{~min}$ followed by the DLS measurement (five independent measurements of acquisition time $\mathrm{t}=300 \mathrm{~s}$ ). Herein, the $T_{C P}$ corresponds to the onset of the increase of the scattered light intensity.

\section{(Co)polymerization kinetic screening}

Stock solutions $(15 \mathrm{~mL})$ of polymerization mixtures were prepared in the glove box by mixing monomer(s) (total concentration $4 \mathrm{M})$ and MeOTs $\left([\mathrm{M}]_{0}:[\mathrm{MeOTs}]_{0}=100: 1\right)$ with acetonitrile and divided over a series of $2.5 \mathrm{~mL}$ microwave vials, each filled with $0.8 \mathrm{~mL}$ of the stock solution. The microwave vials were sealed and heated at $140{ }^{\circ} \mathrm{C}$ for a predetermined amount of time, after which samples were taken for GC and SEC analysis

For the determination of copolymerization parameters, polymerization mixtures containing varying amount of comonomers (Table S1 and S2, total concentration $4 \mathrm{M}$ ) and MeOTs $\left([\mathrm{M}]_{0}:[\mathrm{MeOTs}]_{0}=100: 1\right)$ in acetonitrile were sealed in $2.5 \mathrm{~mL}$ microwave vials and heated at $140{ }^{\circ} \mathrm{C}$ for $5 \mathrm{~min}(\mathrm{MeOzi} / \mathrm{PrOx})$, respectively $6 \mathrm{~min}(\mathrm{MeOzi} / \mathrm{BuOx})$. Afterwards the sample for GC characterization was taken and the polymerization mixture was terminated with $1 \mathrm{M}$ solution of potassium hydroxide ( 3 eqiv. of initial MeOTs amount). The polymerization solvent and most of the remaining monomers were evaporated under reduced pressure, the crude polymer was dissolved in dichloromethane, precipitated in ice-cold diethyl ether and dried under high vacuum. The polymer composition $(F)$ was determined by ${ }^{1} \mathrm{H}$ NMR spectroscopy from the integrals of the side-chain peaks (Table S1 and S2). The copolymerization parameters $\mathrm{r}_{\text {MeOzi, }}$, $\mathrm{r}_{\text {PrOx }}$ and $\mathrm{r}_{\mathrm{BuOx}}$ were calculated using equations $\mathrm{S} 1$ and $\mathrm{S} 2$ (for the explanation of terms see footnote of Table S1). 


$$
\begin{aligned}
& F_{1}=\frac{r_{1} f_{1}^{2}+f_{1} f_{2}}{r_{1} f_{1}^{2}+2 f_{1} f_{2}+r_{2} f_{2}^{2}} \\
& \eta=\left(r_{1}+\left(\frac{r_{2}}{\alpha}\right)\right) \xi-\left(\frac{r_{2}}{\alpha}\right)
\end{aligned}
$$

The distribution of the repeating units along the copolymer chain was calculated using the Skeist model (equations S3 and S4)

$$
\begin{gathered}
X=1-\left(\frac{f_{1}}{f_{1}^{0}}\right)^{a}\left(\frac{f_{2}}{f_{2}^{0}}\right)^{b}\left(\frac{f_{1}^{0}-d}{f_{1}-d}\right)^{c} \\
\bar{F}_{1}=\frac{f_{1}^{0}-f_{1}(1-X)}{X}
\end{gathered}
$$

where $X$ is total monomer conversion, $f_{1}{ }^{0}$ and $f_{2}{ }^{0}$ are initial molar fractions of monomer 1 respectively $2 ; f_{1}$ and $f_{2}$ are instant monomer fractions of monomer 1 respectively 2 and $\bar{F}_{1}$ is the cumulative molar fraction of repeating unit 1 in cthe opolymer.

\section{Synthesis of gradient copolymers}

The copolymers were synthesized using the same protocol as used for the determination of copolymerization parameters, with monomer feed ratio and polymerization times given in Table S3 and S4. By this method, PMeOzi-grad-PBuOx (MB1-4), respectively PMeOzi-gradPPrOx (MP1-7) were synthesized. For full characterization see Figures S6-S13 and Tables S3-

\section{S4.}

\section{Determination of critical micelle concentration $(\mathrm{cmc})$}

Solutions of the copolymers were prepared by direct dissolution in phosphate buffered saline (PBS, $150 \mathrm{mM}, \mathrm{pH}$ 7.4) at a concentration of $1 \mathrm{mg} \mathrm{mL}^{-1}$ and were successively diluted to a concentration of $10^{-5} \mathrm{mg} \mathrm{mL}^{-1}$. Pyrene stock solution $\left(1.2 \times 10^{-4} \mathrm{~mol} \mathrm{~L}^{-1}\right.$ in methanol) was added to each polymer sample, so the final concentration of pyrene in PBS thus was $610^{-}$ ${ }^{7} \mathrm{~mol} \mathrm{~L}^{-1}$. Fluorescence spectra of the samples were recorded with Cary Eclipse spectrophotometer (under stirring) equipped with a Varian Cary Temperature Controller, equilibrated for $10 \mathrm{~min}$ at $37{ }^{\circ} \mathrm{C}$, using excitation at $333 \mathrm{~nm}$. The ratio of the fluorescence emission intensities at $372 \mathrm{~nm}\left(\mathrm{I}_{1}\right)$ and $383\left(\mathrm{I}_{3}\right)$ was plotted against the polymer concentration. 
The cmc was determined as the intersection between the plateau at $\mathrm{d}\left(\mathrm{I}_{1} / \mathrm{I}_{3}\right) / \mathrm{dc} \approx 0$ and the tangent of the curve where the $\mathrm{I}_{1} / \mathrm{I}_{3}$ ratio decreased with an increasing copolymer concentration.

\section{Encapsulation of curcumin}

Curcumin was loaded into the MB4 micelles using a nanoprecipitation technique. MB4 (40 mg) and curcumin $(5 \mathrm{mg})$ were dissolved in anhydrous ethanol $(10 \mathrm{~mL})$ and injected at room temperature into the rapidly stirred distilled water $(20 \mathrm{~mL})$. After stirring at room temperature for $60 \mathrm{~min}$, the amount of solvents was reduced in vacuo to $5 \mathrm{~mL}$, refilled with distilled water to $10 \mathrm{~mL}$ and the insoluble drug was removed by centrifugation at $8000 \mathrm{rpm}$. The supernatant was filtered through $0.22 \mu \mathrm{m}$ syringe filter and freeze-dried. The amount of loaded curcumin was determined by UV-VIS spectroscopy in methanol at $424 \mathrm{~nm}$ using curcumin calibration. Then, the drug loading was calculated as $\mathrm{DL}=\mathrm{m}_{\text {cur }} /\left(\mathrm{m}_{\mathrm{MB} 4}+\mathrm{m}_{\mathrm{cur}}\right) \times 100 \%$, where $\mathrm{m}_{\text {cur }}$ and $\mathrm{m}_{\mathrm{MB} 4}$ are the weight amounts of the solubilized curcumin and MB4 copolymer in the dispersion and the entrapment efficiency $\mathrm{EE}=\mathrm{m}_{\text {cur }} / \mathrm{m}_{\text {cur added }} \times 100 \%$, where $\mathrm{m}_{\text {cur added }}$ is the initial amount of curcumin added to polymer solution.

\section{In vitro cytotoxicity of MB1-4}

Polymer stock solutions were prepared in PBS and a concentration range was prepared by serial dilution. SKOV-3 cells were seeded into 96-well titer plates (10000 cells per well, suspended in $200 \mu \mathrm{L}$ of culture medium) and incubated overnight. Next, $50 \mu \mathrm{L}$ of sample, DMSO (positive control $=0 \%$ viability) or PBS (negative control $=100 \%$ viability), was added to the cells, followed by $24 \mathrm{~h}$ of incubation. Subsequently, the medium was aspirated, and the cells were washed with $250 \mu \mathrm{L}$ of PBS. After aspiration, $100 \mu \mathrm{L}$ of MTT working solution was added and the cells were incubated for $2.5 \mathrm{~h}$. Finally, the MTT working solution was aspirated and the formed purple formazan crystals were dissolved in $50 \mu \mathrm{L}$ of DMSO. Absorbance was determined at $590 \mathrm{~nm}$ using an EnVision Multilabel plate reader. The absorbance of the positive control was used as blank and therefore subtracted from all values. Cell viability (\%) was defined as follows:

$$
\text { cell viability }=\frac{A b s(\text { sample })-A b s(+ \text { control })}{A b s(- \text { control })-A b s(+ \text { control })} \times 100 \%
$$




\section{In vitro cellular uptake of MB1-4}

For microscopy, SKOV-3 cells were plated in WillCo Wells dishes at a density of $5^{*} 10^{3}$ cells per well (suspended in $190 \mu \mathrm{L}$ ) and allowed to adhere overnight. Subsequently, cells were pulsed for 16 hours with micelles, $10 \mu \mathrm{L}$ of $10 \mathrm{mg} / \mathrm{mL}$ R18-labeled copolymers, followed by washing with PBS and fixation with $4 \%$ paraformaldehyde. R18 in pure PBS was used as blank. Prior to imaging, cell nuclei were stained with Hoechst 33342 (according to manufacturer's protocol) and the cell membrane was stained with AlexaFluor488-conjugated phalloidin. Confocal microscopy was carried out on a Leica DMI6000 B inverted microscope equipped with an oil immersion objective (Leica, 40×, NA 0.75) and attached to an Andor DSD2 confocal scanner. Images were processed using the ImageJ software package.

For flow cytometry, a $10 \mathrm{mg} / \mathrm{mL}$ stock solution of rhodamine octadecylamine in ethanol was prepared. Subsequently, $5 \mu \mathrm{L}$ of this solution was diluted to $1 \mathrm{~mL}$ ethanol. $100 \mu \mathrm{L}$ of the rhodamine octadecylamine working solution was added to $1 \mathrm{~mL}$ of polymer solution (10 $\mathrm{mg} \mathrm{mL}^{-1}$ ) in PBS. Formulations were stabilized overnight at room temperature with an open lid to allow evaporation of the ethanol. Finally, excess dye was removed by membrane filtration $(0.450 \mu \mathrm{m})$. Loading of R18 in MB1-4 micelles (Table S4) was determined by UV-VIS spectroscopy in PBS-ethanol mixture (1:1) at $561 \mathrm{~nm}$ using R18 calibration in the same solvent. SKOV-3 cells were seeded into 24-well titer plates ( 250000 cells per well, suspended in $0.9 \mathrm{~mL}$ of culture medium) and incubated overnight to allow cell sedimentation and subsequent adhesion to the bottom of the wells. Next, $100 \mu \mathrm{L}$ of sample (at different dilution) was added to the cells, followed by $24 \mathrm{~h}$ of incubation to allow cellular uptake. After incubation, the wells were aspirated and washed with $1 \mathrm{~mL}$ of PBS. After subsequent aspiration of the wells, $500 \mu \mathrm{L}$ of cell dissociation buffer was added to the cells. The cells were completely detached from the wells after $15 \mathrm{~min}$ of incubation. The cell suspensions were transferred into Eppendorf tubes and immediately centrifuged $\left(350 \mathrm{~g}, 15 \mathrm{~min}, 5^{\circ} \mathrm{C}\right)$. Finally, the supernatant was aspirated and the cell pellets were suspended into $300 \mu \mathrm{L}$ of PBS and kept on ice to maintain cell integrity. FACS was performed on a BD Accuri C6 (BD Biosciences). The data were processed using the FlowJo software package. 


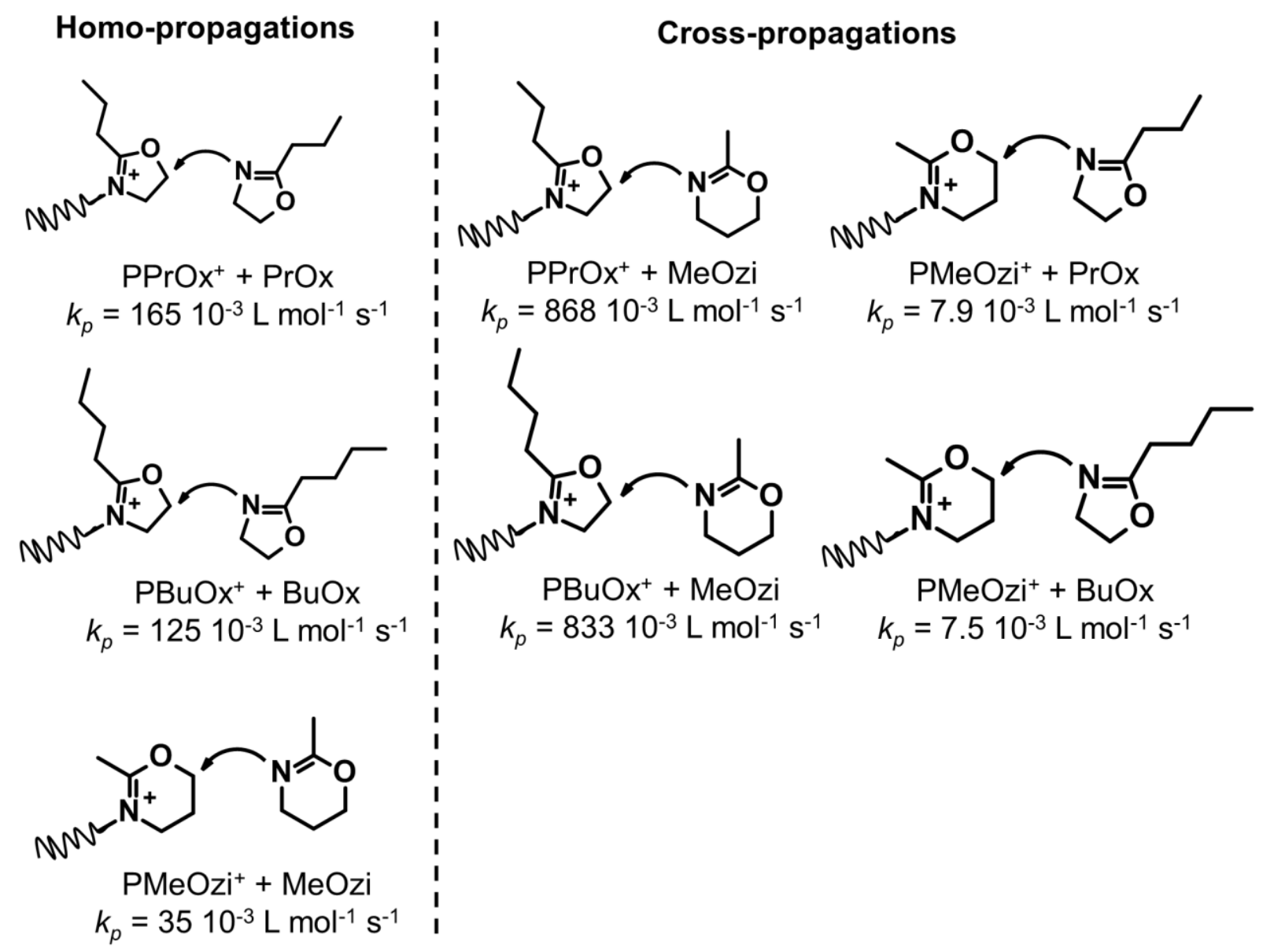

Figure S1. Overview of the measured apparent propagation rate constants $\left(k_{p}\right)$ for individual propagation events. Homo-propagation rate constants were obtained from the homopolymerization kinetics. Cross-propagation rate constants were calculated from copolymerization parameters $(r)$ and homopolymerization rate constants. 

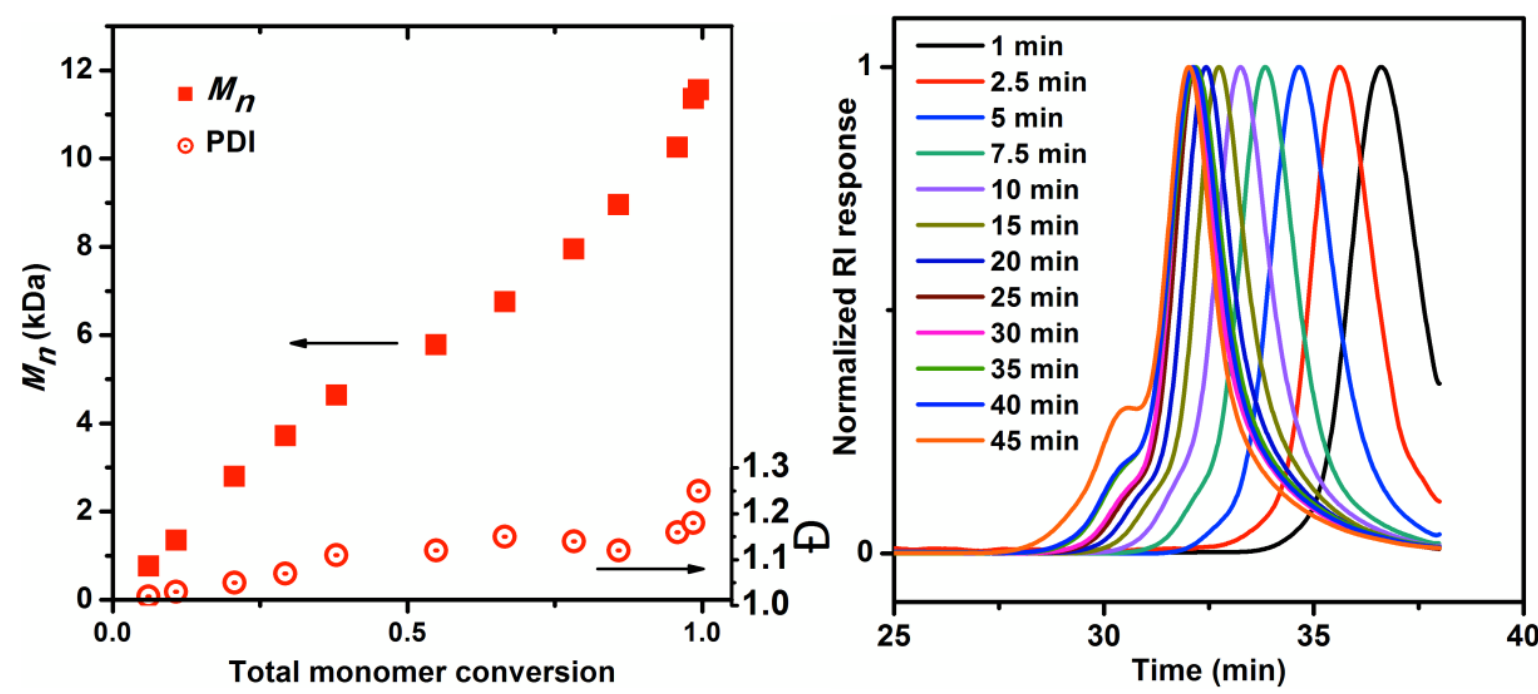

Figure S2. Copolymerization of $\mathrm{MeOzi}$ with $\mathrm{BuOx}$ at $140^{\circ} \mathrm{C}$ in acetonitrile initiated with MeOTs, with initial total monomer concentration $[\mathrm{M}]_{0}=4 \mathrm{M}$ and initial concentrations $[\mathrm{MeOzi}]_{0}:[\mathrm{BuOx}]_{0}:[\mathrm{MeOTs}]_{0}=50: 50: 1$ : The number-average molecular weights and dispersities plotted vs. total monomer conversion determined by SEC-MALS (left). SEC traces eluted with DMA-LiCl (right).
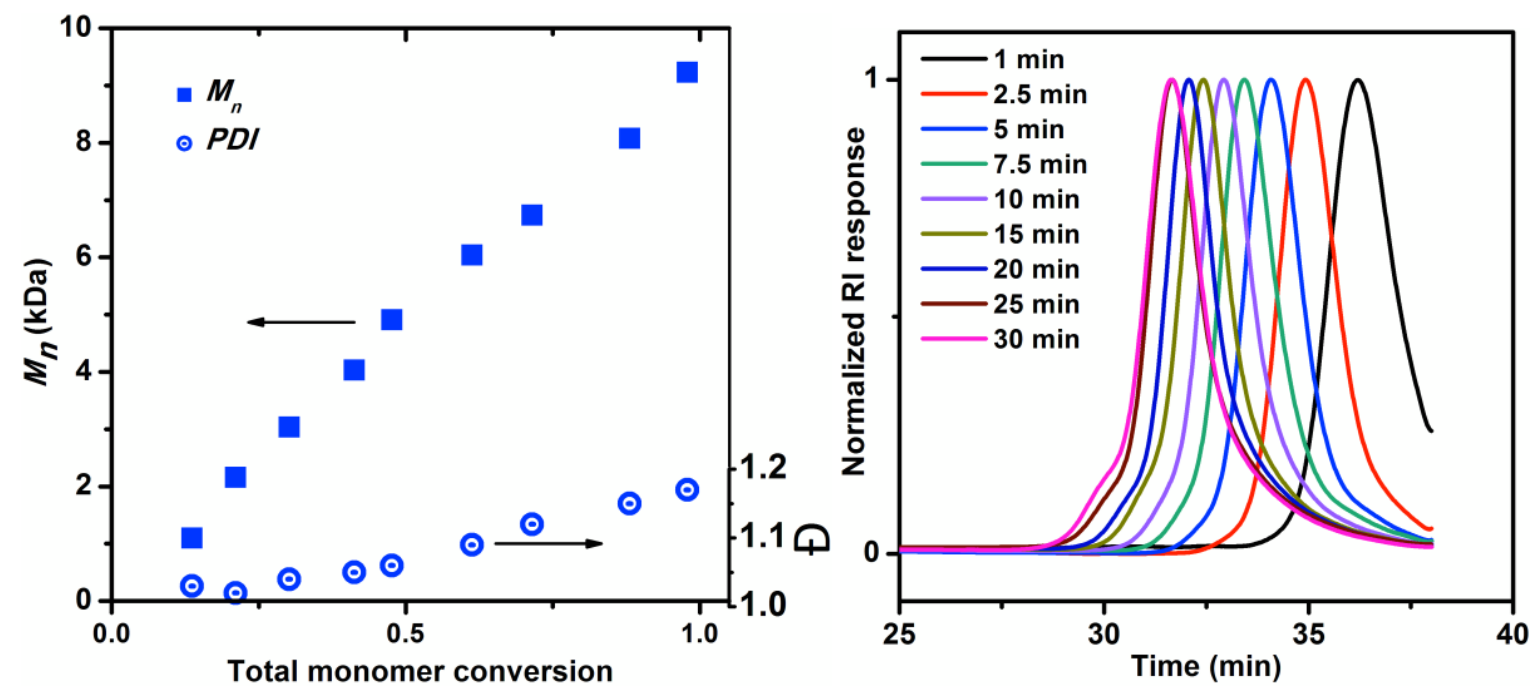

Figure S3. Copolymerization of $\mathrm{MeOzi}$ with $\mathrm{PrOx}$ at $140^{\circ} \mathrm{C}$ in acetonitrile initiated with MeOTs, with initial total monomer concentration $[\mathrm{M}]_{0}=4 \mathrm{M}$ and initial concentrations $[\mathrm{MeOzi}]_{0}:[\mathrm{PrOx}]_{0}:[\mathrm{MeOTs}]_{0}=50: 50: 1$ : The number-average molecular weights and dispersities plotted vs. total monomer conversion determined by SEC-MALS (left). SEC traces eluted with DMA-LiCl (right). 
Table S1. Parameters used for calculation of monomer reactivity ratios in $\mathrm{MeOzi} / \mathrm{BuOx}$ copolymerization at $140^{\circ} \mathrm{C}$ in acetonitrile initiated with MeOTs, with initial total monomer concentration $[\mathrm{M}]_{0}=4 \mathrm{M},[\mathrm{M}]_{0}:[\mathrm{MeOTs}]_{0}=100: 1$ and reaction time of 6 min.

\section{Conversion}

\begin{tabular}{cccccccccc} 
Entry & $\mathrm{f}_{\mathrm{MeOzi}}{ }^{\mathrm{a}}$ & $\mathrm{F}_{\mathrm{MeOzi}}{ }^{\mathrm{b}}$ & $\zeta_{\mathrm{MeOzi}}{ }^{\mathrm{c}}$ & $\zeta_{\mathrm{PrOx}}{ }^{\mathrm{d}}$ & $\mathrm{Z}^{\mathrm{e}}$ & $\mathrm{G}^{\mathrm{f}}$ & $\mathrm{H}^{\mathrm{g}}$ & $\eta^{h}$ & $\xi^{i}$ \\
\hline 1 & 0.91 & 0.965 & 0.335 & 0.085 & 4.578 & 5.804 & 1.316 & 4.084 & 0.926 \\
\hline 2 & 0.80 & 0.92 & 0.381 & 0.103 & 4.399 & 2.387 & 0.594 & 3.410 & 0.849 \\
\hline 3 & 0.69 & 0.88 & 0.381 & 0.094 & 4.832 & 1.311 & 0.314 & 3.122 & 0.748 \\
\hline 5 & 0.59 & 0.83 & 0.422 & 0.101 & 5.145 & 0.755 & 0.184 & 2.601 & 0.636 \\
\hline 6 & 0.49 & 0.775 & 0.509 & 0.115 & 5.811 & 0.421 & 0.102 & 2.025 & 0.491 \\
\hline 7 & 0.39 & 0.687 & 0.553 & 0.131 & 5.740 & 0.208 & 0.067 & 1.208 & 0.387 \\
\hline 8 & 0.30 & 0.563 & 0.576 & 0.149 & 5.305 & 0.054 & 0.046 & 0.359 & 0.302 \\
\hline 9 & 0.10 & 0.175 & 0.898 & 0.367 & 4.997 & -0.158 & 0.008 & -1.381 & 0.074
\end{tabular}

${ }^{\mathrm{a}}$ Molar fraction of MeOzi in monomer feed. ${ }^{\mathrm{b}}$ Molar fraction of MeOzi in polymer determined by ${ }^{1} \mathrm{H}$ NMR. ${ }^{\mathrm{c}}$ Monomer conversion determined by GC, ${ }^{\mathrm{d}} \mathrm{Z}=\log \left(1-\zeta_{\mathrm{MeOzi}}\right) / \log \left(1-\zeta_{\text {PrOx }}\right),{ }^{\mathrm{d}} \mathrm{G}^{\prime}=$ $(\mathrm{F}-1) / \mathrm{Z}$ where $\mathrm{F}=\mathrm{F}_{\text {MeOzi }} / \mathrm{F}_{\text {PrOx }},{ }^{\mathrm{g}} \mathrm{H}^{\star}=\mathrm{F} / \mathrm{Z}^{2},{ }^{\mathrm{h}} \eta=\mathrm{G}^{\prime} /\left(\mathrm{H}^{\star}+\alpha\right)$ where $\alpha=\left(\mathrm{H}^{\star}{ }_{\max } \mathrm{H}^{\star}{ }_{\text {min }}\right)^{0.5},{ }^{\mathrm{i}} \xi=\mathrm{H}^{\star} /($ $\left.\mathrm{H}^{\circ}+\alpha\right)$.
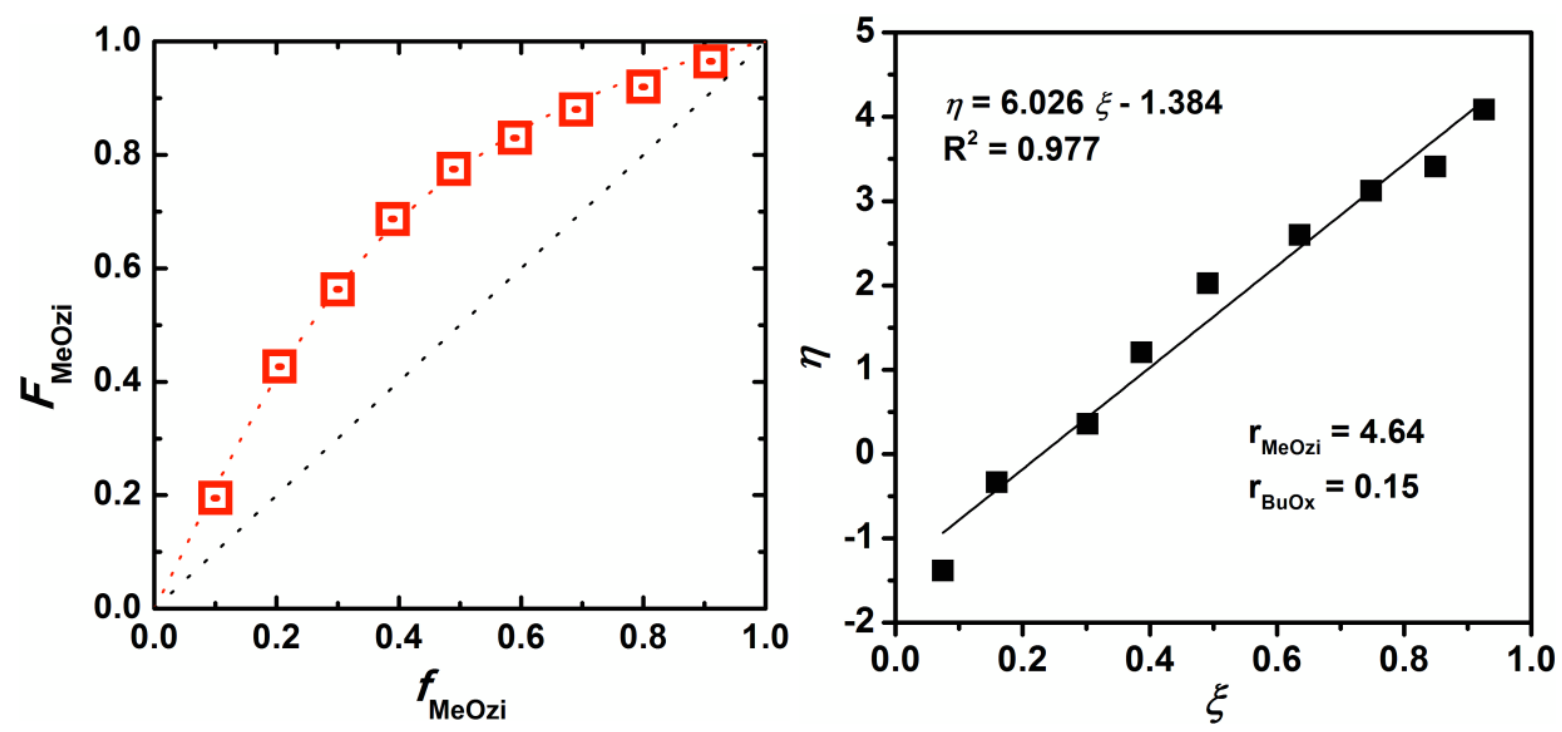

Figure S4. Copolymerization of MeOzi with PrOx: Mayo-Lewis plot (left), Extended KelenTudos plot (right). 
Table S2. Parameters used for calculation of monomer reactivity ratios in $\mathrm{MeOzi} / \mathrm{PrOx}$ copolymerization at $140^{\circ} \mathrm{C}$ in acetonitrile initiated with MeOTs, with initial total monomer concentration $[\mathrm{M}]_{0}=4 \mathrm{M},[\mathrm{M}]_{0}:[\mathrm{MeOTs}]_{0}=100: 1$ and reaction time of $5 \mathrm{~min}$.

\section{Conversion}

\begin{tabular}{cccccccccc} 
Entry & $\mathrm{f}_{\mathrm{MeOzi}}{ }^{\mathrm{a}}$ & $\mathrm{F}_{\mathrm{MeOzi}}{ }^{\mathrm{b}}$ & $\zeta_{\mathrm{MeOzi}}{ }^{\mathrm{c}}$ & $\zeta_{\mathrm{PrOx}}{ }^{\mathrm{d}}$ & $\mathrm{Z}^{\mathrm{e}}$ & $\mathrm{G}^{\prime \mathrm{f}}$ & $\mathrm{H}^{` \mathrm{~g}}$ & $\eta^{h}$ & $\xi^{i}$ \\
\hline 1 & 0.9 & 0.970 & 0.320 & 0.079 & 4.696 & 6.602 & 1.451 & 4.239 & 0.932 \\
\hline 2 & 0.8 & 0.927 & 0.429 & 0.118 & 4.467 & 2.632 & 0.639 & 3.530 & 0.858 \\
\hline 3 & 0.7 & 0.881 & 0.413 & 0.114 & 4.414 & 1.456 & 0.381 & 2.987 & 0.782 \\
\hline 4 & 0.6 & 0.820 & 0.424 & 0.122 & 4.228 & 0.841 & 0.255 & 2.330 & 0.706 \\
\hline 5 & 0.5 & 0.761 & 0.456 & 0.126 & 4.530 & 0.480 & 0.155 & 1.842 & 0.593 \\
\hline 7 & 0.4 & 0.677 & 0.585 & 0.163 & 4.941 & 0.222 & 0.086 & 1.1552 & 0.447 \\
\hline 8 & 0.3 & 0.579 & 0.687 & 0.188 & 5.597 & 0.067 & 0.044 & 0.449 & 0.293 \\
\hline 9 & 0.1 & 0.157 & 0.969 & 0.507 & 4.891 & -0.166 & 0.008 & -1.461 & 0.068
\end{tabular}

${ }^{\mathrm{a}}$ Molar fraction of MeOzi in monomer feed. ${ }^{\mathrm{b}}$ Molar fraction of MeOzi in polymer determined by ${ }^{1} \mathrm{H}$ NMR. ${ }^{\mathrm{c}}$ Monomer conversion determined by GC, ${ }^{\mathrm{d}} \mathrm{Z}=\log \left(1-\zeta_{\mathrm{MeOzi}}\right) / \log \left(1-\zeta_{\text {PrOx }}\right),{ }^{\mathrm{d}} \mathrm{G}^{\prime}=$

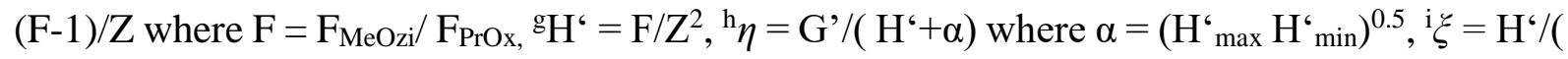
$\left.\mathrm{H}^{6}+\alpha\right)$.
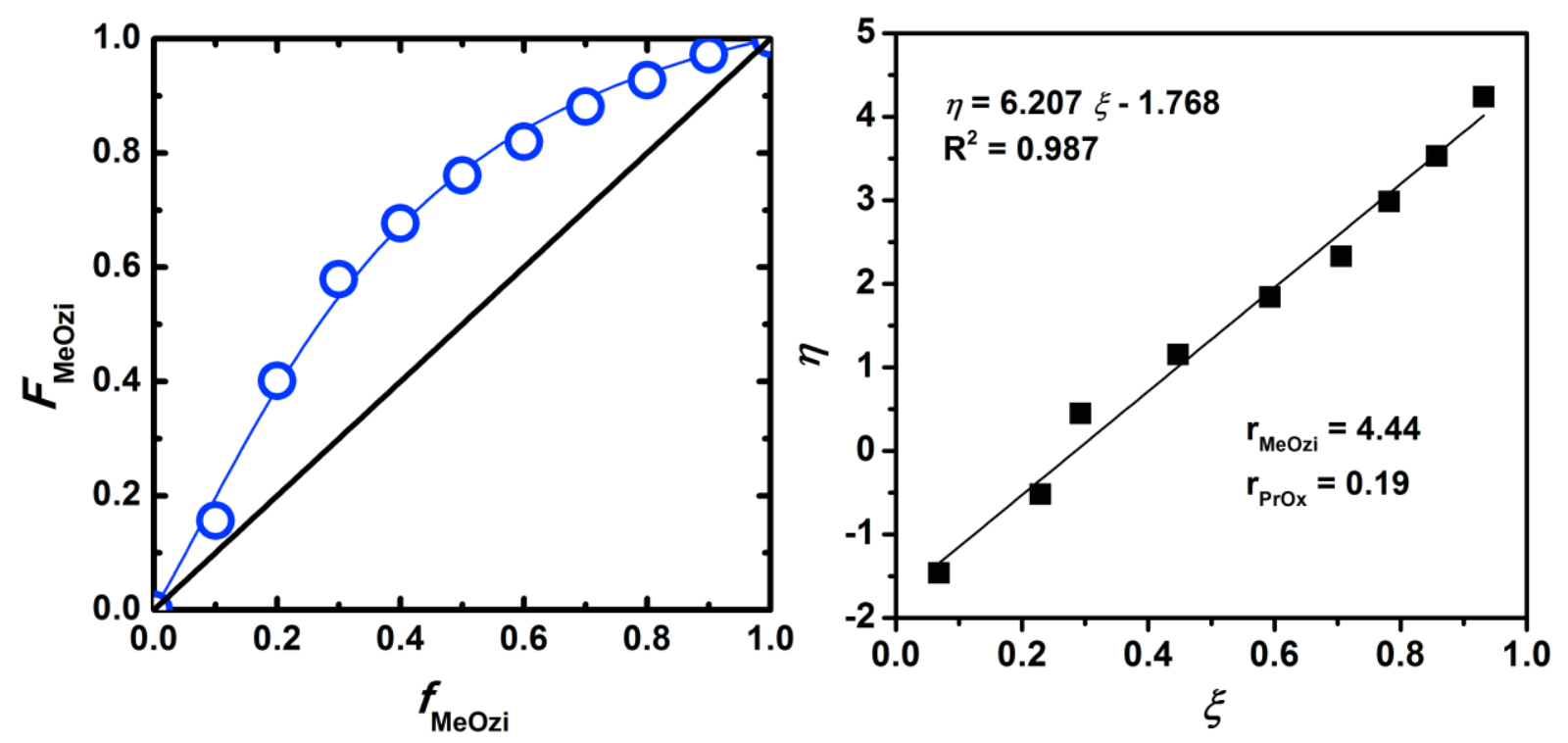

Figure S5. Copolymerization of MeOzi with PrOx: Mayo-Lewis plot (left), Extended KelenTudos plot (right). 
Table S3. Reaction conditions and characteristics of PMeOzi-grad-PBuOx copolymers.

\begin{tabular}{cccccccc}
\hline Polymer & {$[\mathrm{MeOzi}]_{0}:[\mathrm{BuOx}]_{0}{ }^{\mathrm{a}}$} & $\begin{array}{c}\text { Time } \\
(\mathrm{min})^{\mathrm{b}}\end{array}$ & $\begin{array}{c}\mathrm{F}_{\mathrm{MeOzi}} \\
(\%)^{\mathrm{c}}\end{array}$ & $\begin{array}{c}M_{n} \\
\left(\mathrm{~kg} \mathrm{~mol}^{-1}\right)^{\mathrm{d}}\end{array}$ & $\begin{array}{c}D^{\mathrm{d}} \\
(\mathrm{nm})^{\mathrm{e}}\end{array}$ & $\begin{array}{c}\mathrm{cmc} \\
\left(\mathrm{mg} \mathrm{L}^{-1}\right)^{\mathrm{f}}\end{array}$ \\
\hline MB1 & $50: 50$ & 35 & 53 & 9.07 & 1.12 & 33.5 & 6.0 \\
\hline MB2 & $60: 40$ & 40 & 62 & 9.41 & 1.10 & 27.8 & 7.2 \\
\hline MB3 & $70: 30$ & 45 & 72 & 8.22 & 1.13 & 25.1 & 7.1 \\
\hline MB4 & $80: 20$ & 70 & 81 & 8.82 & 1.25 & 6.8 & n.d. \\
\hline
\end{tabular}

anitial monomer feed ratio. ${ }^{\mathrm{b}}$ Polymerization time. ${ }^{\mathrm{c}} \mathrm{MeOzi}$ fraction in copolymer determined by ${ }^{1} \mathrm{H}$ NMR. ${ }^{\mathrm{d}}$ Determined by SEC-MALS using DMA/LiCl as an eluent. ${ }^{\mathrm{e}} \mathrm{Hydrodynamic}$ diameter determined by DLS $\left(\mathrm{c}_{\mathrm{pol}}=2 \mathrm{mg} \mathrm{mL}^{-1}\right)$. ${ }^{\mathrm{f}}$ Critical micelle concentration.

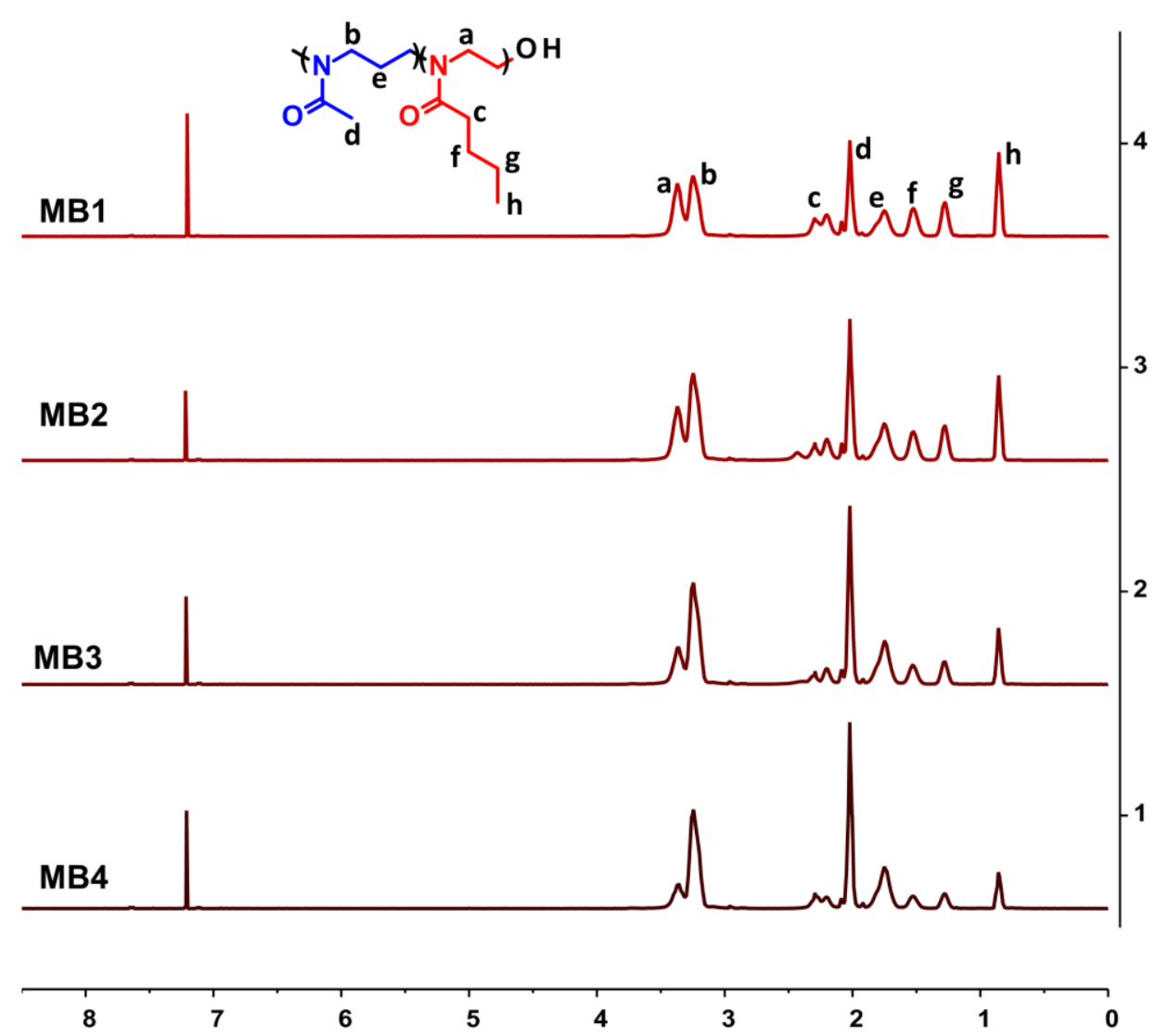

Figure S6. ${ }^{1} \mathrm{H}$ NMR spectra of PMeOzi-grad-PBuOx copolymers MB1-4 in $\mathrm{CDCl}_{3}$. 


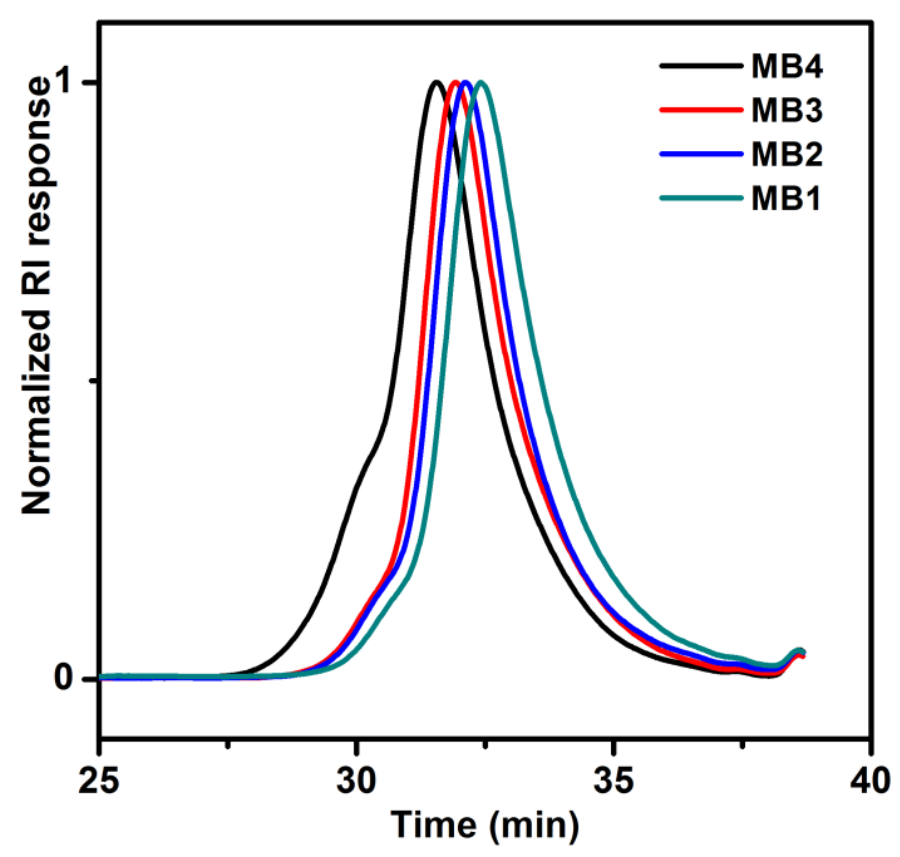

Figure S7. SEC traces of PMeOzi-grad-PBuOx copolymers eluted with DMA-LiCl.

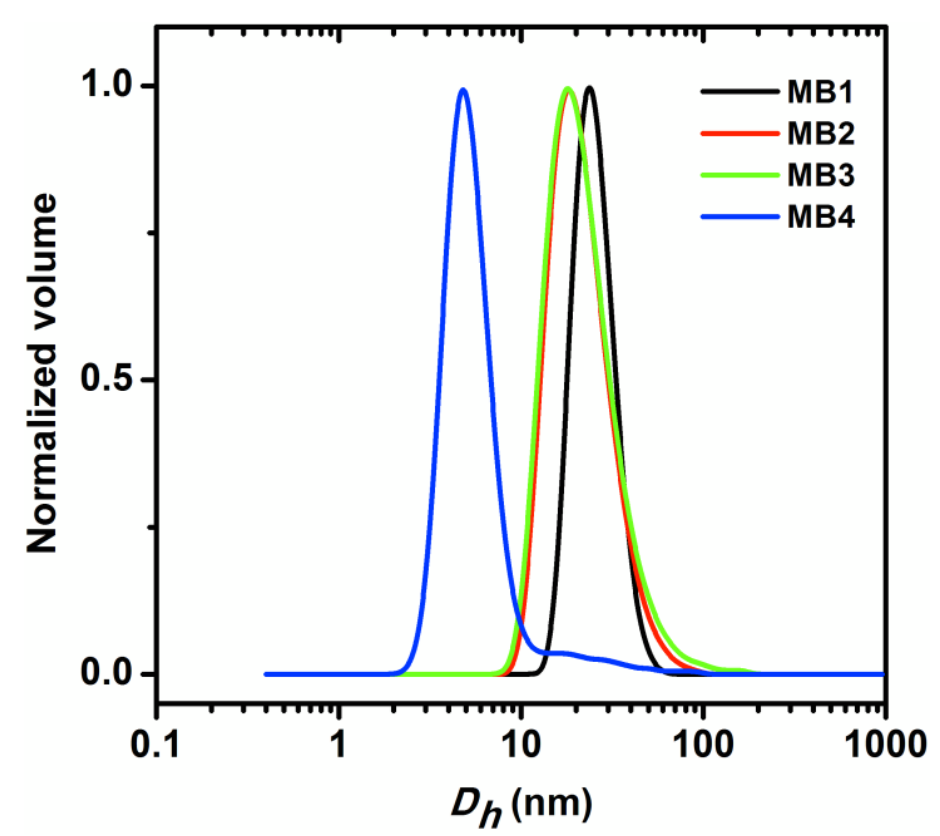

Figure S8. Hydrodynamic diameters $\left(D_{h}\right)$ of PMeOzi-grad-PBuOx copolymers MB1-4 in PBS $\left(\mathrm{pH}=7.4, \mathrm{c}_{\mathrm{pol}}=2 \mathrm{mg} \mathrm{mL}^{-1}\right)$. 

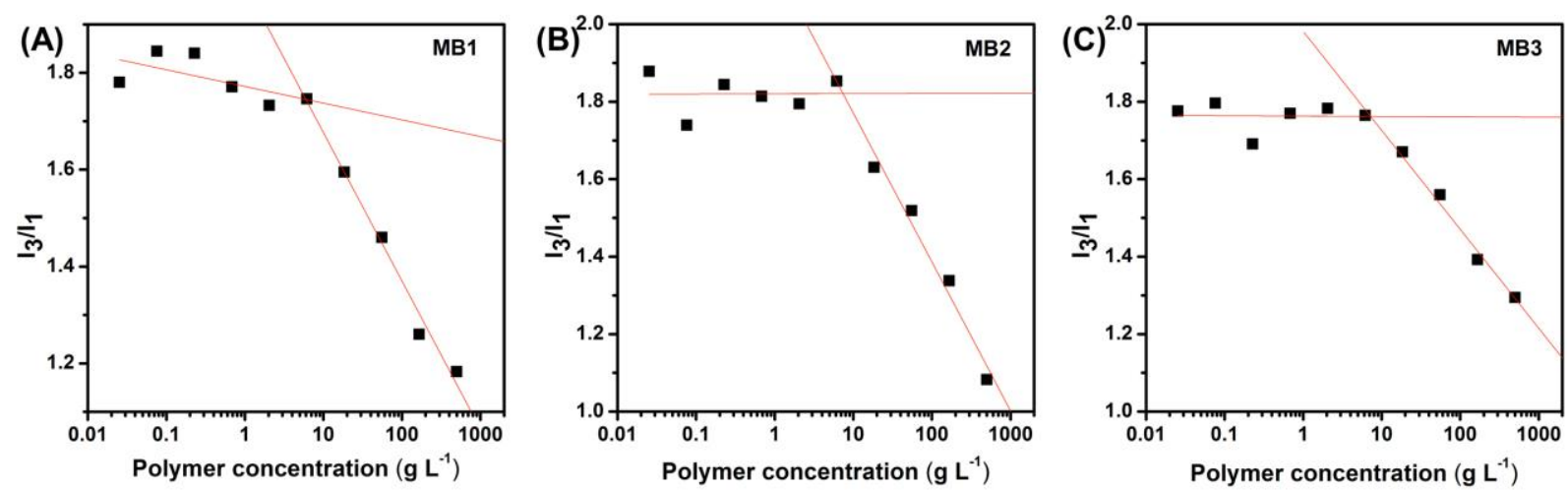

Figure S9. Dependence of the $I_{3} / I_{1}$ pyrene fluorescence ratio on MB1-3 copolymer concentration, used for the determination of critical micelle concentrations $(\mathrm{cmc})$.

Table S4. Characteristics of the MB1-4 micelles loaded with octadecylrhodamine (R18).

\begin{tabular}{ccc}
\hline Polymers & $\begin{array}{c}\text { R18 loading } \\
(\mathrm{wt} \%)^{\mathrm{a}}\end{array}$ & $\begin{array}{c}\text { Encapsulation } \\
\text { efficiency }(\%)^{\mathrm{a}}\end{array}$ \\
\hline MB1 & 0.042 & 83.8 \\
\hline MB2 & 0.038 & 75.7 \\
\hline MB3 & 0.039 & 78.0 \\
\hline MB4 & 0.034 & 67.6
\end{tabular}

${ }^{\mathrm{a}}$ Determined by UV/VIS spectroscopy.

Table S5. Reaction conditions and characteristics of PMeOzi-grad-PPrOx copolymers.

\begin{tabular}{cccccccc}
\hline Polymer & $\begin{array}{c}{[\mathrm{MeOzi}]_{0}:} \\
{[\mathrm{PrOx}]_{0}{ }^{\mathrm{a}}}\end{array}$ & $\begin{array}{c}\text { Time } \\
(\mathrm{min})^{\mathrm{b}}\end{array}$ & $\begin{array}{c}\mathrm{F}_{\text {MeOzi }} \\
(\%)^{\mathrm{c}}\end{array}$ & $\begin{array}{c}M_{n} \\
\left(\mathrm{~kg} \mathrm{~mol}^{-1}\right)^{\mathrm{d}}\end{array}$ & $\bigoplus^{d}$ & $\mathrm{~T}_{\mathrm{cp} 1}\left({ }^{\circ} \mathrm{C}\right)^{\mathrm{e}}$ & $\mathrm{T}_{\mathrm{cp} 2}\left({ }^{\circ} \mathrm{C}\right)^{\mathrm{f}}$ \\
\hline MP1 & $20: 80$ & 20 & 22 & 10.1 & 1.08 & 29.5 & 30.7 \\
\hline MP2 & $30: 70$ & 25 & 31 & 10.0 & 1.11 & 32.0 & 34.3 \\
\hline MP3 & $35: 65$ & 30 & 36 & 10.4 & 1.11 & 33.0 & 40.3 \\
\hline MP4 & $40: 60$ & 35 & 45 & 10.7 & 1.16 & 37.0 & 50.8 \\
\hline MP5 & $50: 50$ & 45 & 51 & 10.5 & 1.17 & 41.0 & 60.7 \\
\hline MP6 & $60: 40$ & 50 & 61 & 10.4 & 1.20 & 47.0 & 74.4 \\
\hline MP7 & $85: 15$ & 55 & 89 & 7.81 & 1.17 & $-\mathrm{g}$ & $-\mathrm{g}$ \\
\hline
\end{tabular}

anitial monomer feed ratio. ${ }^{\mathrm{b}}$ Polymerization time. ${ }^{\mathrm{c}} \mathrm{MeOzi}$ fraction in copolymer determined by ${ }^{1} \mathrm{H}$ NMR. ${ }^{\mathrm{d}}$ Determined by SEC-MALS using DMA/LiCl as an eluent. ${ }^{\mathrm{e} C l o u d-p o i n t}$ temperature determined by DLS as the onset of scattering intensity increase. ${ }^{\mathrm{f}}$ Cloud-point 
temperature determined by turbidimetry. 'Sample soluble as single chains in the whole temperature range $\left(15-85^{\circ} \mathrm{C}\right)$.

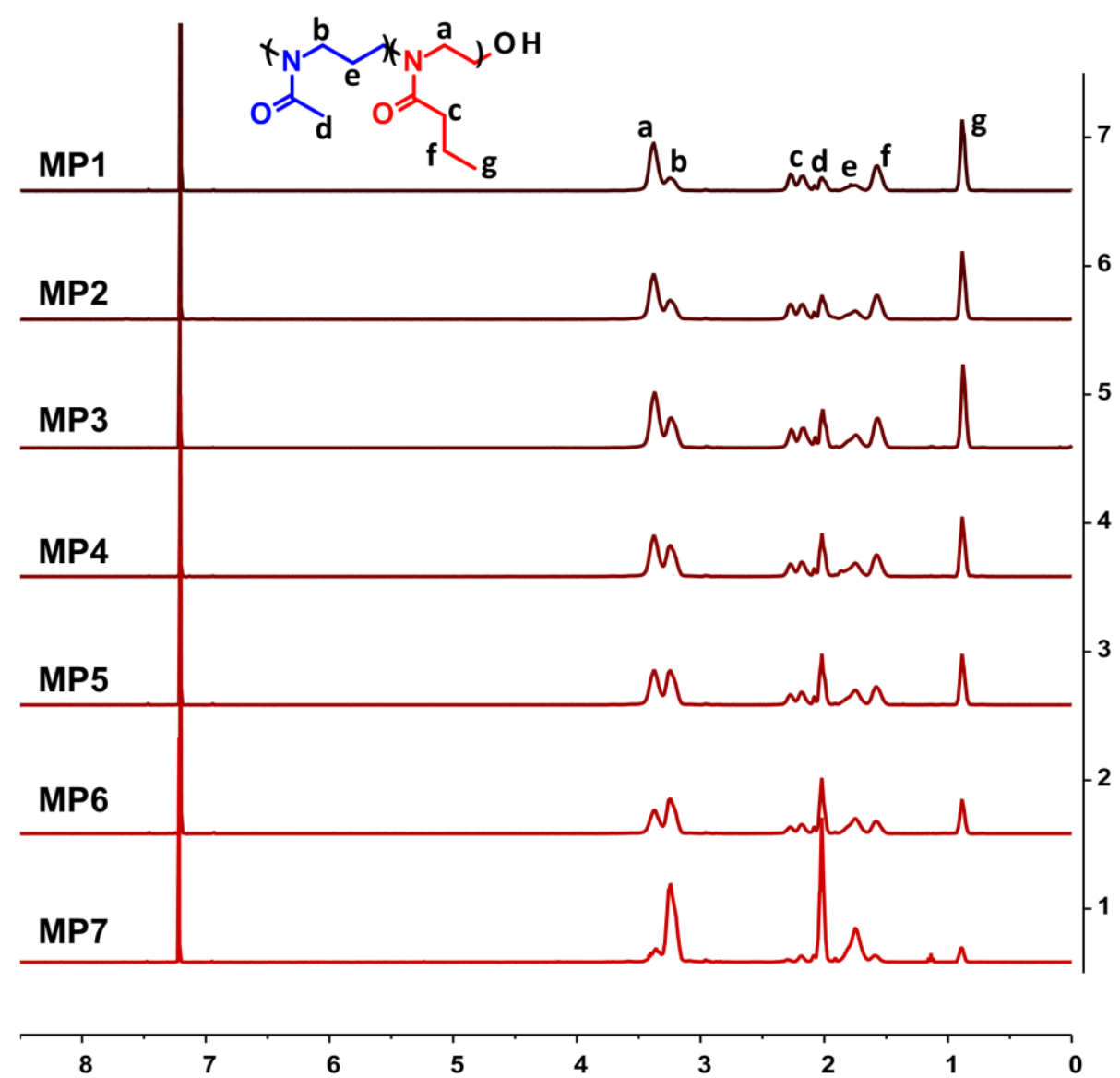

Figure S10. ${ }^{1} \mathrm{H}$ NMR spectra of PMeOzi-grad-PPrOx copolymers MP1-7 in $\mathrm{CDCl}_{3}$. 


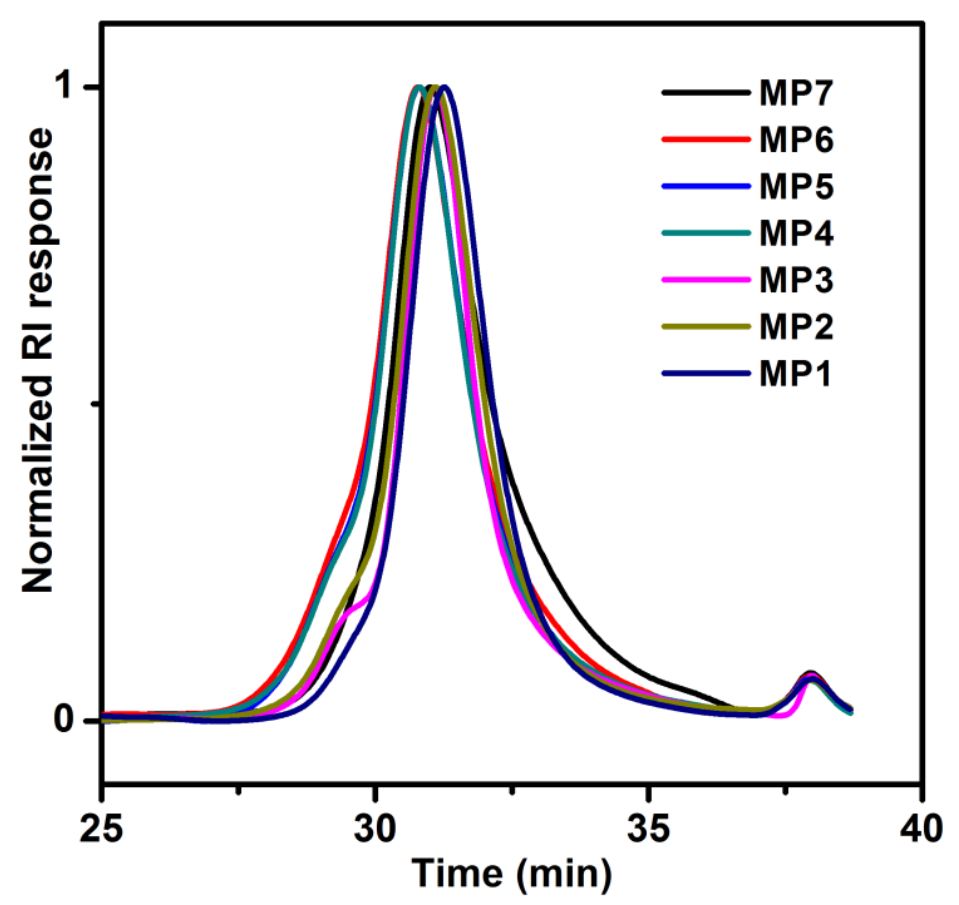

Figure S11. SEC traces of PMeOzi-grad-PPrOx copolymers eluted with DMA-LiCl. 

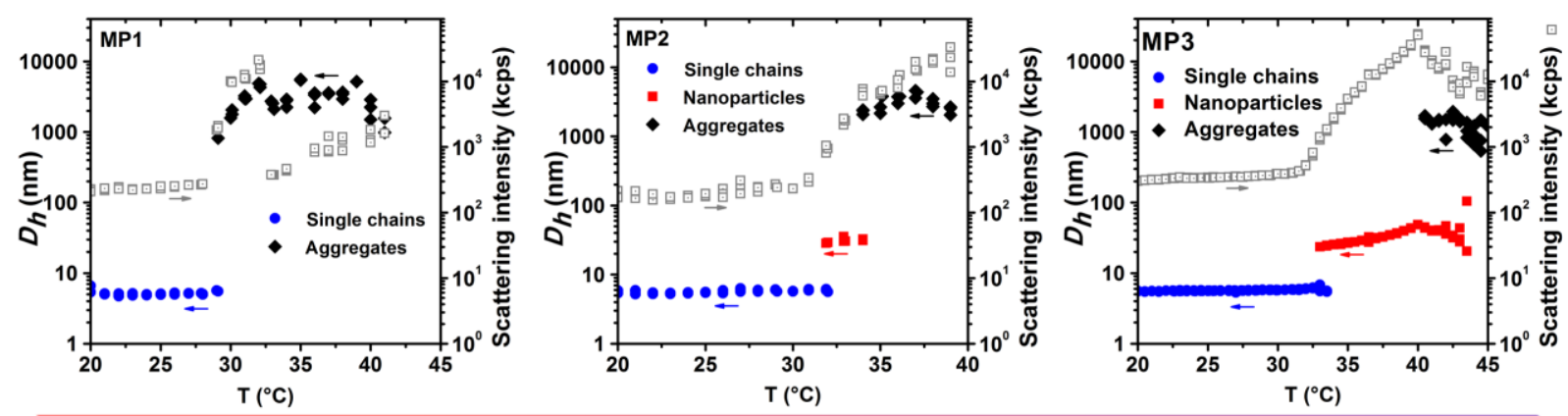

\section{Increasing MeOzi content}
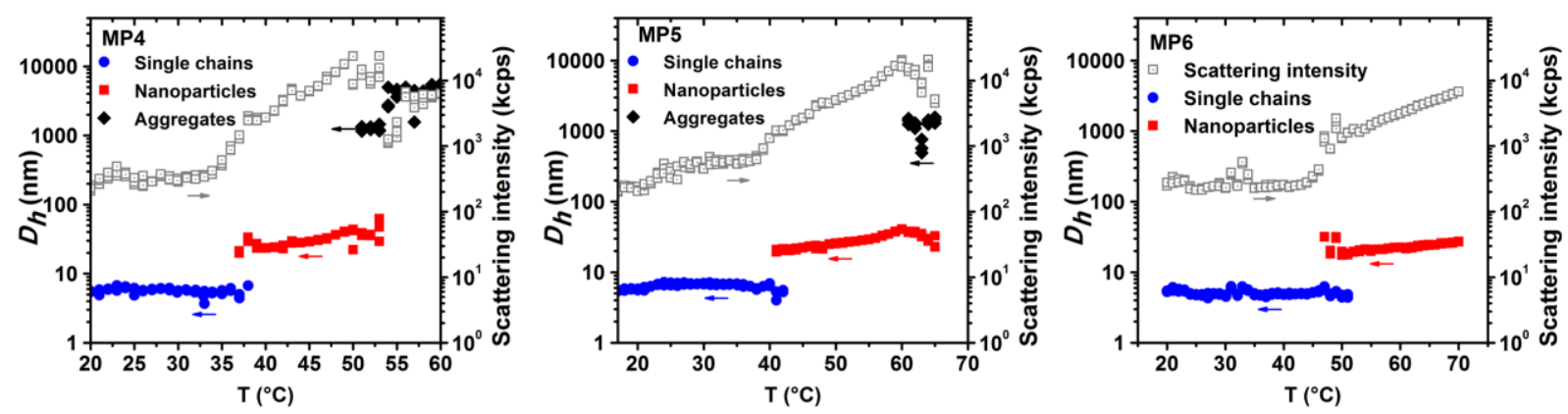

Increasing MeOzi content

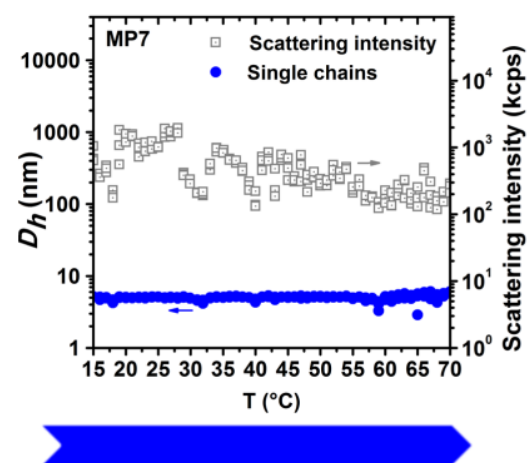

Figure S12. Temperature dependence of the hydrodynamic diameter and scattering light intensity of the PMeOzi-grad-PPrOx copolymers MP1-7 in PBS ( $\left.\mathrm{pH}=7.4, \mathrm{c}_{\mathrm{pol}}=5 \mathrm{mg} \mathrm{mL}^{-1}\right)$. 


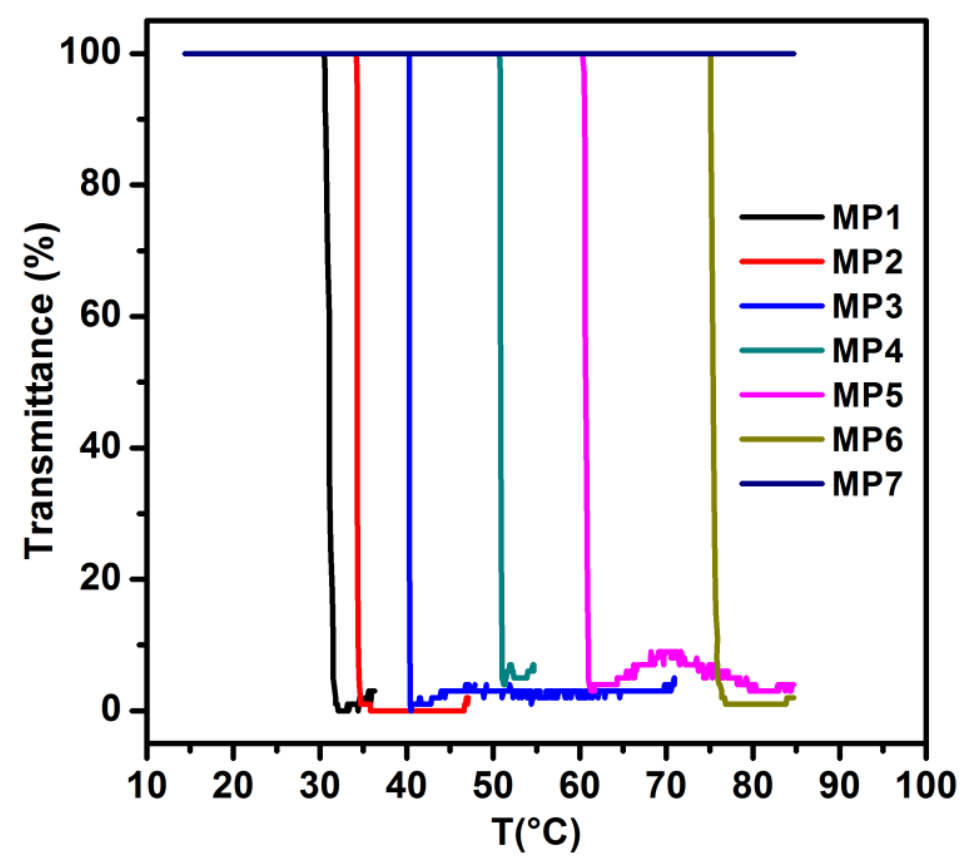

Figure S13. LCST behavior of PMeOzi-grad-PPrOx copolymers MP1-7 in PBS (pH = 7.4, $\left.\mathrm{c}_{\mathrm{pol}}=5 \mathrm{mg} \mathrm{mL}^{-1}\right)$ as determined turbidimetry.

\section{References}

1. (a) Bloksma, M. M.; Paulus, R. M.; van Kuringen, H. P.; van der Woerdt, F.;

Lambermont-Thijs, H. M.; Schubert, U. S.; Hoogenboom, R., Thermoresponsive Poly (2oxazine) s. Macromol. Rapid Commun. 2012, 33 (1), 92-96; (b) Bloksma, M. M.; Weber, C.; Perevyazko, I. Y.; Kuse, A.; Baumgärtel, A.; Vollrath, A.; Hoogenboom, R.; Schubert, U. S., Poly (2-cyclopropyl-2-oxazoline): from rate acceleration by cyclopropyl to thermoresponsive properties. Macromolecules 2011, 44 (11), 4057-4064. 